\title{
An Improved Nonlinear PID Control Method for a Class of Servo
}

\author{
Lingling Wang ${ }^{1, a}$, Qiang Zhou ${ }^{2, b}$, Aijun Zhou ${ }^{3}$, Junwei Lei ${ }^{1}$ \\ ${ }^{1}$ Department of Control, Naval Aeronautical and Astronautical University, Yantai, 264001, China \\ ${ }^{2}$ Department of Scientific Research, Naval Aeronautical and Astronautical University, Yantai, \\ 264001, China \\ ${ }^{3}$ Department of Navigation, Dalian Naval Academy, Dalian, 116018, China \\ aemail:lingling0711@163.com, bemail:zhouqiang1024@126.com
}

keywords: Servo; Nonlinear; PID

\begin{abstract}
For the second order simplified model of servo system, the traditional PID control method is used to design the feedback controller of the servo system, and the PID control parameters are adjusted to make the output stable and smooth without chatter. On this basis, an improved nonlinear PID control method is introduced, which makes the response speed of the servo system faster and the output not overshoot or flutter, in line with the needs of engineering applications.
\end{abstract}

\section{Introduction}

The servo system is a heading automatic control system to ensure the flight of missiles and other aircraft in a given course. The performance of the servo system directly affects the safety performance, economic performance and military performance of the aircraft [1-3]. PID control is a widely used control method in servo systems for many years [2-5]. The traditional PID control is realized by proportional, integral and differential control of the deviation between the desired signal and the actual output. However, the linear mapping becomes a defect of its control, such as the contradiction between dynamic performance and static performance, control performance and robust performance. Therefore, many scholars proposed to use fuzzy control, variable structure, neural networks and other methods of control [6-8], while these algorithms can achieve better control in the simulation results, but the practical application of system reliability is difficult to guarantee. Therefore, this paper designs a nonlinear PID control algorithm based on PID control, which can be applied to the typical model of missile servo system. The simulation results show that the nonlinear PID control algorithm can obviously improve the performance of the servo system.

\section{Model Description}

A simplified model of a missile servo system can be described as a differential equation 1[9-10].

$\dot{y}_{a}=-\frac{1}{T} y_{a}+\frac{1}{T} u$

$\dot{y}=y_{a}$

Where $T$ is the inertial time constant, and $T=0.06 \mathrm{~s}, u$ is the control input, $y_{a}$ is the rotational angular velocity, $y$ is the output. The correlation between $y_{a}$ and $u$ can be described as the transfer function 2.

$\frac{y_{a}}{u}=\frac{1}{T S+1}$

Where $S$ is the differential Operators in transfer Function.

So the whole servo system model can be described as follows figure 1 . 


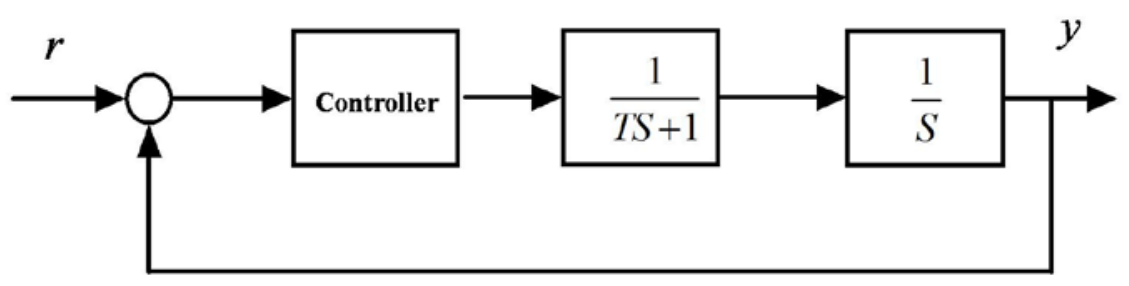

Figure 1 Schematic diagram of servo system

The control objective is to design the controller such that the output position signal $y$ of the servo system tracks the desired signal $r$ [11].

\section{PID Controller Design}

Define the error variable $e=r-y$, and design the PID controller as the formula 3 .

$u=k_{1} e+k_{2} \dot{e}+k_{3} \int e d t$

Where $k_{1}, k_{2}, k_{3}$ are the proportion, differential and integral coefficient respectively, $\dot{e}$ is the derivative of error, and $\int e d t$ is the integration of error. Generally suppose $r$ is the constant step signal, and $r=5 / 57.3$, then we get the formula 4 .

$\dot{e}=\dot{r}-\dot{y}=-y_{a}$

\section{Simulation Results of PID Control}

Set the control parameters as $\mathrm{k} 1=5, \mathrm{k} 2=0.2, \mathrm{k} 3=0.5$. Simulation result is shown in figure 2 .

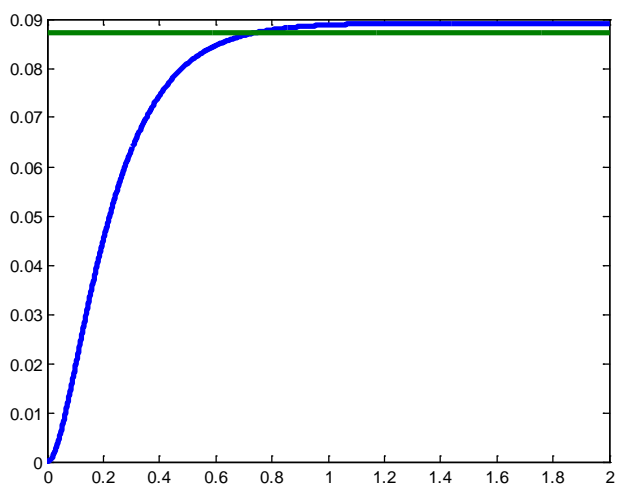

Figure 2 Servo output response curve

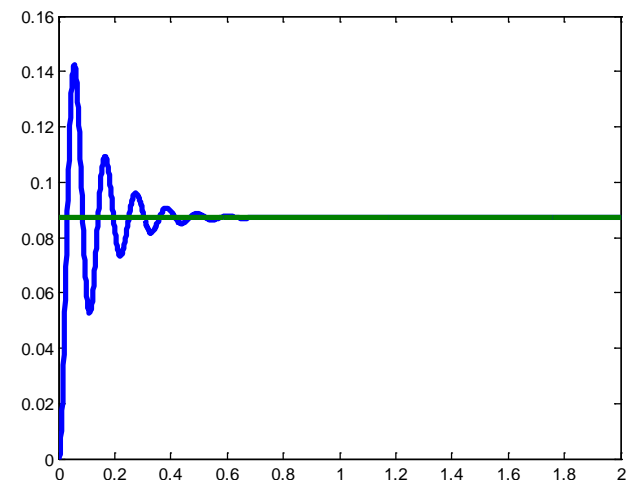

Figure 3 Servo output response curve

In order to make the system response faster and steady-state error smaller, we increase the control coefficient and the system response curve is shown in figure 3. In this case the system oscillates more, so we can increase the damping coefficient and system response curve is shown in figure 4.

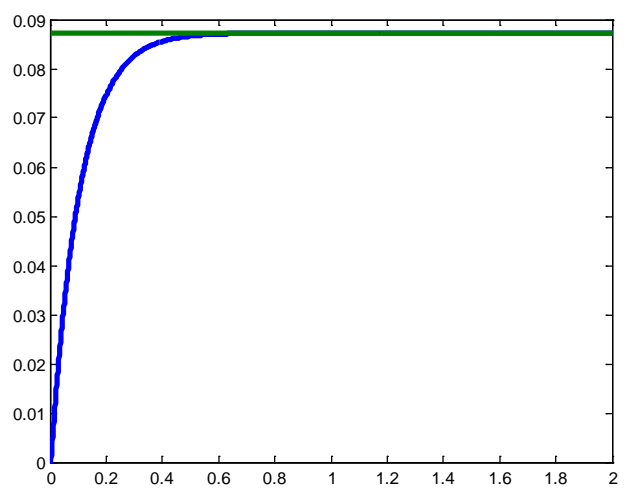

Figure 4 Servo output response curve

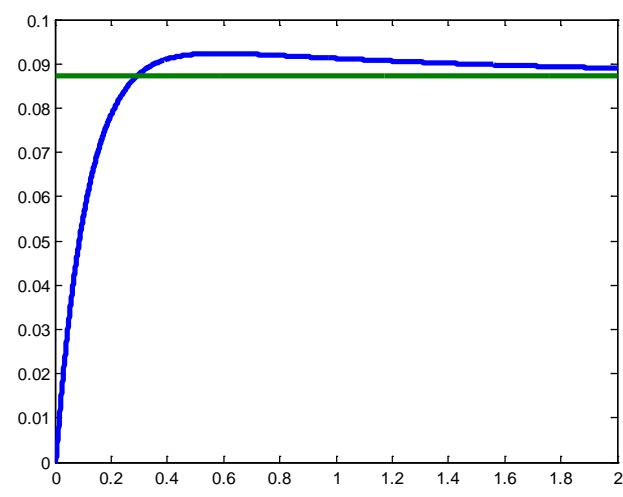

Figure 5 Servo output response curve 
As shown in figure 4, the system response speed is still not fast enough, so try to increase the integral coefficient, and the system response curve is shown in figure 5.

\section{Nonlinear PID control algorithm}

Nonlinear PID control algorithm is proposed as the formula 5, based on the above PID control.

$$
u=k_{1} e+k_{2} \dot{e}+k_{3} \int e d t+k_{4} e^{3 / 5}+k_{5} e^{5 / 3}
$$

Set the parameters as $\mathrm{k} 1=200, \mathrm{k} 2=20, \mathrm{k} 3=150, \mathrm{k} 4=50$, and simulation results are shown in figure 6 and figure 7.

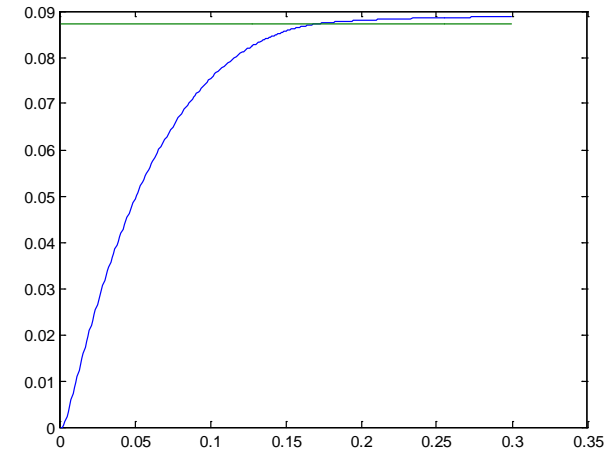

Figure 6 Servo output response curve

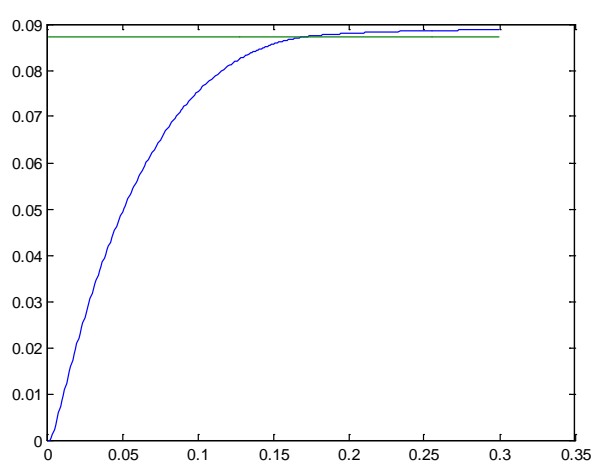

Figure 7 Control value response curve

From the simulation results, it can be seen that the improved nonlinear PID control algorithm makes the servo response speed from $25 \mathrm{~ms}$ to $15 \mathrm{~ms}$. At the same time the control volume of the servo system is also very smooth with no flutter, in line with engineering needs.

\section{Conclusions}

In this paper, the PID control is designed and simulated for a simplified model of servo system is. Then an improved nonlinear PID control method is used to control and simulate the servo system. It can be concluded from the simulation curves that the nonlinear PID control algorithm can improve the response speed of the system and improve the smoothness of the system.

\section{Reference}

[1] Lin Liu, Sub-system of Flight Control System [M], Beijing, National Defense Industry Press, 2003 (in Chinese)

[2] Yongyi Lin, Xiaoqin Chen, Tao Zhou, Design of Automatic Servo for Ship Based on Nonlinear PID [J], Ship and Electronic Technology, 2009, 29(6):46-49 (in Chinese)

[3] Feiyao Dong, Humin Lei, Hua Zeng ,Application of Nonlinear PID Control in Missile Electromechanical Actuator System [J], Tactical Missile Technology, 2009(6):54-57 (in Chinese)

[4] Teng Zhang, Xiaoyu Ni, Design of control system for civil aircraft steering gear [J], Man-Machine Management and Development, 2012(3):14-17(in Chinese)

[5] Yinze Wang, Xunbo Li, Simulation and Analysis of Servo System of Unmanned Aerial Vehicle Electromechanical Actuator [J], Mechanical Research and Application, 2010(5): 36-38 (in Chinese)

[6] Yongliang Huang, Xiaochun Lu, Fuzzy PID control of ship steering gear [J] .Manufacturing \& Manufacturing Technology, 2009(7):27-29 (in Chinese)

[7] Yu Chen, Hongwei Wang, Guoshan Xie, Study on Application of Sliding Mode Variable Structure Control Technology in Ship Autopilot [J], Ship and Electronic Technology, 2008, 28(6):351-353(in Chinese) 
[8] Yang Chong, Ke Zhang, Jingyu Wang, A Design and Realization of Servo Control Algorithm Based on Fuzzy ADRC [J], Journal of Northwestern Polytechnical University, 2011, 29(2):217-222 (in Chinese)

[9] Jianbo Sun, Shiying Gong, Yahui Dong, Simulation of permanent magnet brushless DC motor speed control system [J], Servo technology, 2001, 2(34): 20-23 (in Chinese)

[10] Man Zeng, Xiaoli Xiong, Wenge Ding, A Design of Typical Digital Brushless Electric Steering Gear [J], Journal of North University of China Natural Science Edition, 2011, 32(6):751-757 (in Chinese)

[11] Can Wang, Ruiqing Ma, Bo Tan, Development of DC Servo Actuator Servo System [J] Micro motor, 2008, 36(10):25-27 (in Chinese) 